\title{
Concepciones docentes sobre la calidad de los procesos de enseñanza y aprendizaje de la educación preescolar
}

\author{
Teachers' Conceptions on the Quality of the Teaching and Learning Process in Early \\ Childhood Education
}

\section{Concepções dos professores sobre a qualidade dos processos de ensino e aprendizagem da educação infantil}

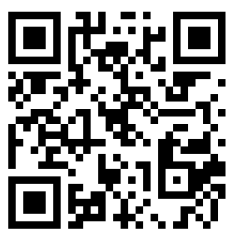
monicavr@um.es

Mónica Vallejo-Ruiz Universidad de Murcia

Murcia, España

Ana Torres-Soto Universidad de Murcia

Murcia, España

ana.t.s@um.es

https://orcid.org/0000-0003-0832-8580

Recibido • Received • Recebido: 10 / 12 / 2018

Corregido • Revised • Revisado: 07 / 05 / 2020

Aceptado • Accepted • Aprovado: 29 / 07 / 2020

\begin{abstract}
Resumen: Este trabajo ofrece una revisión sobre las concepciones de docentes con respecto a la calidad de los procesos de enseñanza y aprendizaje en la etapa de educación preescolar. Siendo ese el principal objetivo del estudio, se realizó una investigación de carácter cuantitativo y de corte descriptivo y correlacional, utilizando como instrumento de medida el cuestionario. Se recogió información sobre cinco dimensiones del proceso de enseñanza y aprendizaje: contexto y características del alumnado, programación, clima de aula, recursos educativos y medios didácticos, y evaluación. Dicho cuestionario se administró a una muestra de 105 docentes de educación preescolar de una de las comunidades autónomas españolas; concretamente la comunidad autónoma de la Región de Murcia (España). Los resultados muestran que el personal docente vincula calidad de la enseñanza-aprendizaje a clima de aula y a recursos y medios utilizados. Asimismo, considera imprescindible que las familias se impliquen en el proceso educativo y que la programación y actividades diseñadas contemplen las necesidades e intereses del alumnado.
\end{abstract}

Palabras claves: Concepciones docentes; calidad; proceso de enseñanza y aprendizaje; educación preescolar. 
http://doi.org/10.15359/ree.24-3.11

http://www.una.ac.cr/educare

educare@una.ac.cr

\begin{abstract}
This paper reviews teachers' conceptions of the quality of teaching and learning processes in preschool education. Since this is the study's main objective, a quantitative, descriptive, and correlational research design was used, utilizing a questionnaire as a measurement instrument. Information was collected on five dimensions of the teaching and learning process: context and student characteristics, programming, classroom atmosphere, educational resources and teaching aids, and assessment. The questionnaire was administered to 105 preschool education teachers from the Autonomous Community of the Region of Murcia (Spain). The outcomes show that teachers link educational quality to the classroom atmosphere and to resources and materials used. Likewise, they consider it essential that families are involved in the educational process and that the programming and activities designed to take the students' needs and interests into account.
\end{abstract}

Keywords: views of teachers; quality; teaching and learning process; Preschool Education.

Resumo: Este trabalho oferece uma revisão das concepções de professores sobre a qualidade dos processos de ensino e aprendizagem na fase pré-escolar. Sendo este o principal objetivo do estudo, foi realizada uma pesquisa quantitativa e descritiva e correlacional, utilizando o questionário como instrumento de medição. Foram coletadas informações sobre cinco dimensões do processo de ensino e aprendizagem: contexto e características dos alunos, programação, clima de sala de aula, recursos educacionais e meios didáticos de ensino e avaliação. Este questionário foi aplicado a uma amostra de 105 professores de educação infantil de uma região espanhola; especificamente a comunidade autônoma da Região de Múrcia (España). Os resultados mostram que o corpo docente vincula qualidade do ensino e aprendizagem ao clima de sala de aula e aos recursos utilizados. Consideram essencialmente importante que as famílias estejam envolvidas no processo educacional e que a programação e as atividades projetadas atendam às necessidades e interesses dos estudantes.

Palavras-chaves: concepções dos professores; qualidade; processo de ensino e aprendizagem; educação infantil.

\title{
Introducción
}

Las investigaciones relativas a las concepciones docentes sobre la calidad de la enseñanzaaprendizaje en la etapa de educación preescolar son bastante escasas (Ruiz de Miguel, 2002; L. Sañudo Guerra y M. I. Sañudo Guerra, 2014). Además de la limitación de estudios, es constatable que los realizados siguen la tendencia actual de analizar la calidad de la enseñanza y aprendizaje desde la elaboración de sistemas de estándares, es decir, desde modelos de enseñanza-aprendizaje ideales presuntamente asociados a la eficacia docente; pero carentes de una contextualización. Esto es, no contemplan el juicio sobre los fines educativos desde una interpretación holística de las concepciones del profesorado en su contexto de práctica docente (Martínez Ortiz et al., 2016). Por consiguiente, la realización de estudios que focalicen su interés en esta etapa educativa y que tomen en consideración a sus docentes, será esencial para construir los cimientos sobre los cuales poder asentar prácticas docentes de calidad en las etapas educativas posteriores. 
De este modo, una vía para avanzar en la mejora de la calidad docente es hacer visibles las prácticas de enseñanza y aprendizaje desde las concepciones del profesorado para romper, con ello, esa privacidad que suele caracterizar la acción educativa. Como es bien sabido, la calidad en educación es un término polisémico que recoge concepciones y usos -en algunas ocasiones- totalmente contrapuestos. Igualmente, es preciso comprender que un aula es, sin lugar a dudas, un entorno complejo por la enorme cantidad de componentes y factores que intervienen y se interrelacionan dentro de ella. Por tanto, se ha de entender el aula desde la perspectiva de ambiente de aprendizaje, en donde las diversas dimensiones o factores que convergen dentro de ella afectan e influyen en los procesos de enseñanza y aprendizaje que se establezcan ahí (Iglesias Forneiro, 2008; Soto-Grant, 2018; TéllezTinoco, 2014). Desde esta óptica, el reconocimiento de la importancia e influencia que los docentes y las docentes conceden a los factores que afectan la calidad de la enseñanza-aprendizaje, motiva poderosamente la realización del estudio que aquí se presenta. Y sobre esta base, los objetivos perseguidos se centran en indagar sobre las concepciones docentes que gravitan alrededor de esa perseguida calidad, al tiempo que describir cómo quedan configuradas tales concepciones con base en diferentes variables independientes previamente definidas que, con toda seguridad, contribuirán a la reflexión para su generalización y permitirán la definición de nuevas líneas de investigación.

\section{Importancia y sentido de definir la calidad educativa}

El tema de la calidad es un concepto arduo complicado de centrar, sobre todo, en educación, a pesar de que se trate con frecuencia en los discursos y agendas educativas. Aguerrondo (2014) indica que la calidad educativa supone un término altamente parcial, es decir, en función de la perspectiva subjetiva de cada individuo puede ser definida y entendida de una forma u otra. Debido a esta implicación, J. F. Miranda Esquer y J. B. Miranda Esquer (2012) exponen que la calidad en educación se encuentra supeditada al momento histórico y social; $y$, por tanto, puede entenderse que al igual que la educación, el concepto de calidad se adapta a los requerimientos particulares de la sociedad en cada época.

Por ende, definir la calidad educativa se vuelve una tarea aún más compleja. En nuestro caso, para el presente estudio, asumiremos que calidad no es igual a perfección; puesto que ninguna acción humana y, por lo tanto, ningún sistema educativo puede ser perfecto, pero sí puede -y debe- aspirar a mejorar (Sammons et al., 2017). De esta manera, se puede entender que una enseñanza de calidad será aquella que permita formar personas capaces de desenvolverse en la sociedad, de la forma más óptima posible y de adaptarse a los sucesivos cambios requeridos.

\section{Concepciones docentes sobre la enseñanza}

Dentro del paradigma del pensamiento del profesorado son numerosos los términos que se han utilizado en la bibliografía científica para definir lo que se entiende por concepciones docentes; términos como: creencias, ideas, representaciones, teorías implícitas, conocimiento práctico, etc. El término concepción encierra una cierta complejidad, pues su definición abarca no 
http://doi.org/10.15359/ree.24-3.11

http://www.una.ac.cr/educare

educare@una.ac.cr

solo referentes cognitivos, sino también contextuales, sociales, históricos y culturales; además del propio conocimiento profesional del personal docente. Un problema no resuelto en la investigación del pensamiento del profesorado es la conexión existente entre conocimientos subjetivos, más o menos vinculados a entramados culturales, con las teorías formales de la formación profesional y que están presentes en la cultura a la cual se pertenece (Martínez Bonafé, 1988).

Desde nuestro punto de vista, es aquí donde radica la importancia de este tipo de estudios. Estudiar las concepciones docentes permitirá desarrollar procesos cognitivos y metacognitivos que faciliten la comprensión de las acciones docentes, y pauten vías para la mejora y la innovación educativa. Algunos estudios sobre las concepciones del personal docente (Barrón Tirado, 2015; Capellini y Rodrigues, 2009; Lucas, 2009; Malcolm, 2006; Solis, 2015) ponen de manifiesto que existe una alta correlación entre las concepciones del profesorado y su práctica docente. Asimismo, permiten incidir en la idea de que el pensamiento docente integra "un cúmulo de teorías implícitas, representaciones, imágenes, suposiciones, nociones, ideas, intenciones, proyectos, supuestos, hipótesis, creencias, actitudes, intereses y valores" (Barrón Tirado, 2015, p. 37) que pueden influir en la toma de decisiones y en la práctica de los procesos de enseñanza y de aprendizaje. De igual modo, Zabalza Beraza (2012) plantea que la práctica educativa está condicionada por las ideas y creencias que los grupos de docentes tienen sobre la enseñanza y el aprendizaje (y sobre la educación en general). Siguiendo estos planteamientos y en coherencia con las sugerencias de Devlin (2006), este estudio parte de la necesidad de analizar dichas concepciones en la medida que estas suponen un primer paso para conocer las prácticas docentes y para analizar, posteriormente, su correspondencia con referentes teóricos sólidos sobre variables que influyen en la calidad educativa en la etapa de educación preescolar.

\section{Método}

\section{Objetivos}

En consideración con este marco de referencia se plantea como objetivo general: Analizar las concepciones del personal docente español con respecto a la calidad de los procesos de enseñanza y aprendizaje en la etapa de educación preescolar (educación infantil en España. En adelante haremos uso de este término para contextualizar los hallazgos encontrados).

A partir de este objetivo general se derivarían los siguientes objetivos específicos:

- Identificar las concepciones del personal docente sobre las dimensiones (contexto y características del alumnado, programación, clima de aula, recursos educativos y medios didácticos y evaluación) y su influencia en la calidad de la enseñanza y aprendizaje en la etapa de educación infantil.

- Determinar el grado de significatividad entre las concepciones docentes sobre la calidad de la enseñanza-aprendizaje y sus características personales y profesionales. 
http://doi.org/10.15359/ree.24-3.11

http://www.una.ac.cr/educare educare@una.ac.cr

\section{Población y muestra}

La muestra de esta investigación estuvo formada por 105 docentes de educación infantil que se distribuyen en centros de educación infantil y primaria de la Región de Murcia (España), tanto públicos como privados o concertados. Fue elegida mediante un sistema de muestreo probabilístico, puesto que todos los sujetos de la población tenían la misma probabilidad de formar parte de la muestra.

La distribución de la muestra por género resulta muy característica y representativa de la etapa educativa analizada. Con una muestra total de 105 sujetos, esta se conforma por 99 mujeres y 6 hombres, es decir, un 94,3\% para el género femenino y un 5,7\% para el género masculino.

Por otro lado, la media de edad de la muestra se encuentra muy cerca del intervalo entre 35 y 45 años (39,4\%). Tanto por encima como por debajo de este intervalo (35-45), docentes de entre 25 y 35, y mayores de 45 años representan un 29,52\% y un 28,57\%, respectivamente. El grueso de la muestra se encuentra, por tanto, distribuido en edades superiores a los 25 años de edad. Así, el personal docente menor de 25 años es muy escaso, con 3 casos excepcionales y un $2,9 \%$ de la muestra total.

En cuanto a la experiencia docente de los sujetos participantes, se detecta que casi el $50 \%$ de la muestra $(49,52 \%)$ se encuentra en las primeras fases de su desarrollo profesional con una experiencia que oscila entre los 5 y 15 años de experiencia con un 49,52\%. El segundo intervalo de experiencia docente más alto es entre 15 y 25 años con un 23,81\% de la muestra total. Según la titularidad del centro en el que imparten clases, la muestra está distribuida, en su gran mayoría, en instituciones públicas con un 81,9\%; por lo que, los centros privados suponen apenas un $18,1 \%$ y dentro de este porcentaje el centro privado-concertado (centros de origen privado que obtienen, además, financiación pública) es el más común con un 16,19\% y un 1,9\% los estrictamente privados.

En cuanto al tamaño de los grupos que cada docente tutela en sus aulas destacan los superiores a 20, con una media de 22,74 estudiantes. Este número es muy heterogéneo entre el personal docente, la ratio de 25 es la más común (22,9\%).

\section{Diseño de la investigación}

Se ha empleado un diseño de tipo cuantitativo y de corte descriptivo tipo encuesta. Se consideró este como el diseño más apropiado, debido a su capacidad para describir propiedades y características importantes del fenómeno en estudio, así como los comportamientos, actitudes u opiniones manifestadas por un grupo de sujetos que forman la población, lo cual permite describir tendencias de un grupo o una población (Hernández Sampieri et al., 2014). Además, admite responder a problemas de investigación en términos descriptivos y de relación de variables, y garantiza el rigor de los datos obtenidos (Latorre et al., 2003). 
http://doi.org/10.15359/ree.24-3.11

http://www.una.ac.cr/educare

educare@una.ac.cr

\section{Instrumento}

Dado que no se encontró un único instrumento que cumpliera con las pretensiones específicas que se buscaban en este estudio, se elaboró un cuestionario ad hoc, no obstante, para el constructo del instrumento se utilizaron varios instrumentos ya creados como referencia importante (Lebrero Baena y Fernández Pérez, 2009; Lera y Oliver, 2002). Partiendo de dichos instrumentos, el cuestionario diseñado está compuesto por 93 ítems que recogen información de cinco dimensiones que inciden en la calidad del proceso de enseñanza y aprendizaje (contexto y características del alumnado, programación, clima de aula, recursos educativos y medios didácticos y evaluación). Para recoger las respuestas del personal docente, se utilizó una escala Likert de 1 a 5 según el grado de valoración que se le otorgue a cada ítem; donde 1 indica nada, 2 poco, 3 regular, 4 bastante y 5 mucho. Es decir, 1 indicaría que ese ítem se considera como un factor o característica que no influye nada en la calidad de los procesos de enseñanza y aprendizaje y un 5 indicaría que sí tiene una gran influencia.

La validación del instrumento se realizó a través de un juicio experto solicitado a 4 especialistas de áreas de conocimiento diferentes que valoraran tanto el contenido del propio instrumento como la fiabilidad de los datos resultantes. El análisis de consistencia interna realizado arrojó un valor de alpha de Cronbach muy aceptable ( $\alpha=0,87)$; lo que implica que existe una alta correlación entre los ítems del cuestionario y que estos miden un mismo constructo.

\section{Procedimiento de recogida y análisis de datos}

El programa utilizado para el análisis de los datos fue el programa SPSS (versión 24) que permite realizar análisis avanzados de datos cuantitativos. Así, con los datos de las encuestas codificados dentro del programa se prosiguió con los diferentes análisis necesarios para cumplir los objetivos de este trabajo.

\section{Resultados y discusión}

Se presentan los resultados en función de los objetivos planteados para este estudio. En primer lugar, se muestran los descriptivos obtenidos en cada una de las dimensiones analizadas, para lo que se acude al análisis de porcentajes. Para facilitar la lectura de los datos y dado que algunas de las dimensiones estudiadas recogían cuantiosa información, la dimensión contexto y características del alumnado se presenta divida en dos subdimensiones: a). Circunstancias personales del alumnado; b). Circunstancias familiares. Por otro lado, la dimensión programación se presenta dividida en los siguientes subdimensiones: c). Planificación de objetivos y contenidos; d). Tareas de aprendizaje; e). Distribución del tiempo; f). Distribución del espacio. Las demás dimensiones se explican sin diferenciar subdimensiones. 
http://doi.org/10.15359/ree.24-3.11

En segundo lugar, se presenta un análisis correlacional de cada una de las dimensiones analizadas y las variables vinculadas a la muestra (género, edad, años de experiencia, etc.).

\section{a. Análisis de las concepciones del personal docente español con respecto a la calidad del proceso de enseñanza y aprendizaje en la etapa de educación infantil}

A la luz de los resultados obtenidos, se puede indicar que el equipo de docentes de educación infantil considera muy importantes todos los ítems planteados en el cuestionario, señalan que todas las dimensiones expuestas afectan la calidad de la enseñanza y el aprendizaje, con puntuaciones medias situadas alrededor de los 4 puntos en una escala del 1 al 5 (Tabla 1).

Tabla 1: Estadísticos descriptivos de las dimensiones analizadas

\begin{tabular}{lccccc}
\hline \multicolumn{1}{c}{ Dimensiones } & N & Mínimo & Máximo & Media & Desv. típ. \\
\hline Circunstancias personales del alumnado & 105 & 2,0 & 5,0 & 3,9 & 0,5 \\
Circunstancias familiares & 105 & 2,0 & 5,0 & 4,1 & 0,6 \\
Planificación de objetivos y contenidos & 105 & 2,6 & 5,0 & 4,3 & 0,5 \\
Tareas de aprendizaje & 105 & 3,2 & 5,0 & 4,3 & 0,4 \\
Distribución del tiempo & 105 & 3,0 & 5,0 & 4,1 & 0,5 \\
Distribución del espacio & 105 & 3,0 & 5,0 & 4,0 & 0,4 \\
Estrategias para gestionar el clima de aula & 105 & 3,3 & 5,0 & 4,5 & 0,3 \\
Medios y recursos educativos & 105 & 3,0 & 5,0 & 4,5 & 0,4 \\
Evaluación de los aprendizajes & 105 & 2,5 & 5,0 & 4,1 & 0,6 \\
\hline
\end{tabular}

Nota: Elaboración propia.

Considerando cada una de las dimensiones de forma individual (atendiendo a los ítems circunscritos a cada dimensión), se han alcanzado los resultados que siguen.

En relación con la dimensión circunstancias personales del alumnado (Figura 1), se considera que la calidad de la enseñanza está muy influenciada por las ausencias reiteradas del estudiantado al aula (50,5\%). Aunque con porcentajes inferiores (entre el 40 y el 50\%) que se distribuyen entre los valores bastante y mucho, el equipo de docentes también considera que las variables cognitivas del alumnado, sus cualidades y su estado físico, condicionan la enseñanza $y$, en consecuencia, su calidad. Se observa, sin embargo, mayor dispersión en las respuestas sobre la influencia de las actividades extraescolares que realizan los alumnos y las alumnas, pues un porcentaje superior de docentes considera que tiene una influencia regular (49,5\%). 
http://doi.org/10.15359/ree.24-3.11

http://www.una.ac.cr/educare

educare@una.ac.cr

Figura 1: Influencia de las circunstancias personales del alumnado en la calidad de la enseñanza y aprendizaje

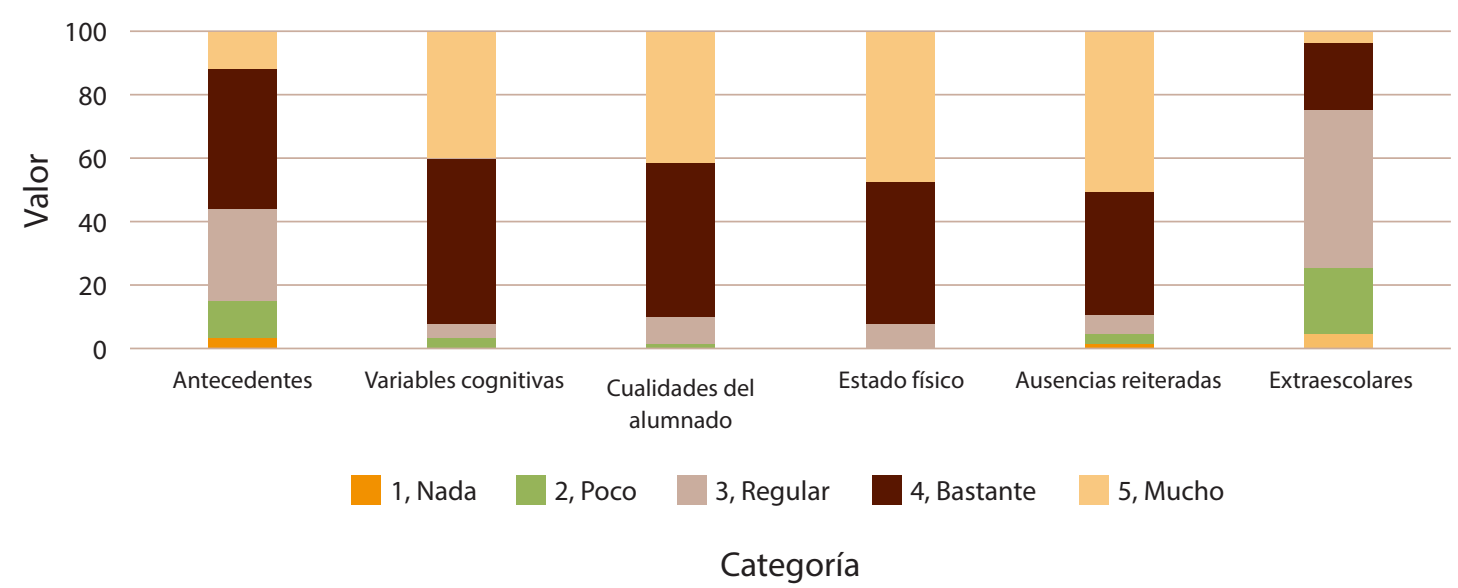

Nota: Elaboración propia.

En relación con la dimensión circunstancias familiares (Figura 2), un porcentaje muy elevado de participantes $(68,6 \%)$ manifiesta que la participación e implicación de los padres y madres influye mucho en la calidad de la enseñanza.

Con porcentajes inferiores, también se consideran variables bastante influyentes, el nivel sociocultural de las familias (55,2\%), las expectativas que estas tienen respecto a sus hijos e hijas $(44,8 \%)$ y el equipamiento cultural del hogar $(53,3 \%)$. Igualmente, la composición de personas que viven en el núcleo familiar resulta una variable influyente, si bien un porcentaje elevado de docentes (36,2\%) cree que influye regular.

Figura 2: Influencia de las circunstancias familiares del alumnado en la calidad de la enseñanza y aprendizaje

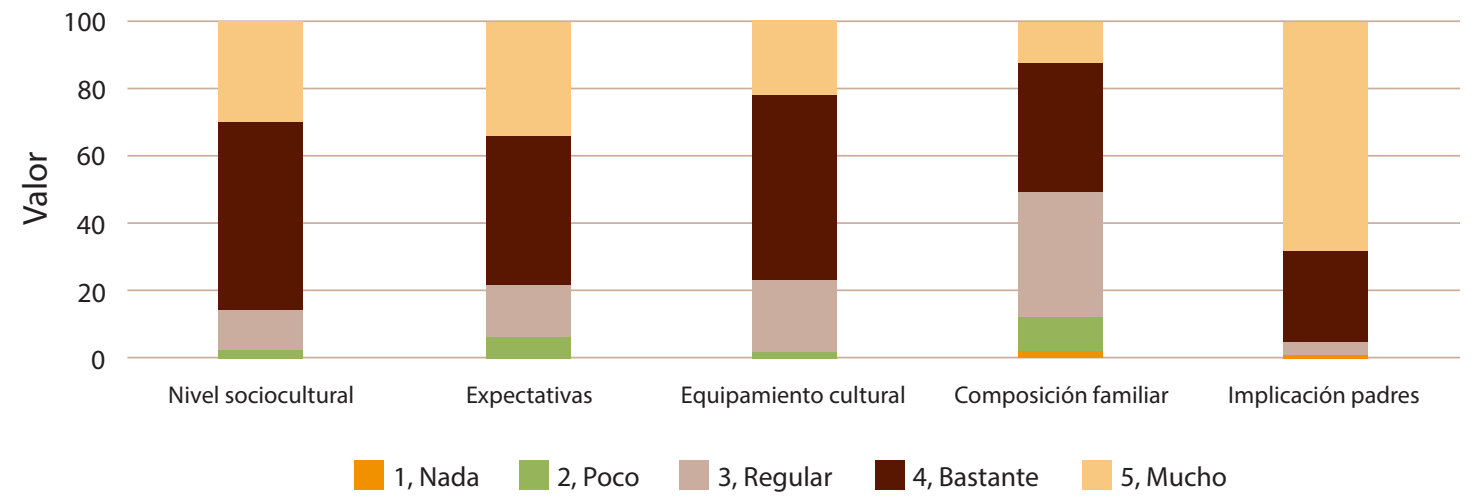

Nota: Elaboración propia.

\section{Categoría}


http://doi.org/10.15359/ree.24-3.11

Además de conocer la importancia del contexto y las características del alumnado, se pretendió valorar la importancia que otorga el profesorado de educación infantil a los elementos relacionados con la dimensión programación de aula. A este respecto, se observa que la planificación de objetivos y contenidos es considerada un aspecto muy influyente en la calidad de la enseñanza; sobre todo, se valora como muy importante adaptar los objetivos a las capacidades del alumnado (69,5\%), así como priorizar, secuenciar y temporalizar esos objetivos $(57,1 \%)$. Asimismo, el equipo de docentes considera que tiene gran influencia (bastante) que se seleccionen esos objetivos -tanto didácticos $(51,4 \%)$, como de etapa $(53,3 \%)$ - de acuerdo con la propuesta curricular y teniendo en cuenta las áreas de conocimiento.

Estos hallazgos ratifican las evidencias de la bibliografía existente sobre la importancia que los cuerpos docentes otorgan al conocimiento de distintos temas sobre diversidad del alumnado y, por consiguiente, la importancia y necesidad de formación docente (Cejudo et al., 2016; Zabalza Beraza, 1996).

Respecto a la dimensión tareas de aprendizaje (Figura 3), el equipo de docentes de educación infantil plantea que tienen una influencia mayor aquellas tareas que conectan con los intereses, necesidades y motivaciones del alumnado (86,7\%). Aceptan que influye mucho la utilización de elementos motivadores para presentar las tareas (80,0\%), así como diseñar actividades introductorias que sirvan de motivación (68,6\%). Del mismo modo, establecen que es muy importante -para que haya calidad en los procesos de enseñanza y aprendizaje- realizar programaciones centradas en el alumnado y en sus necesidades $(61,9 \%)$ y, en consecuencia, que se diseñen actividades que tengan en cuenta los ritmos individuales y respeten la diversidad (75,2\%). Y, como consecuencia de estas concepciones, el 30,5\% de docentes plantea que programar actividades partiendo de las fichas o materiales de un libro de texto influye en una menor calidad en la enseñanza.

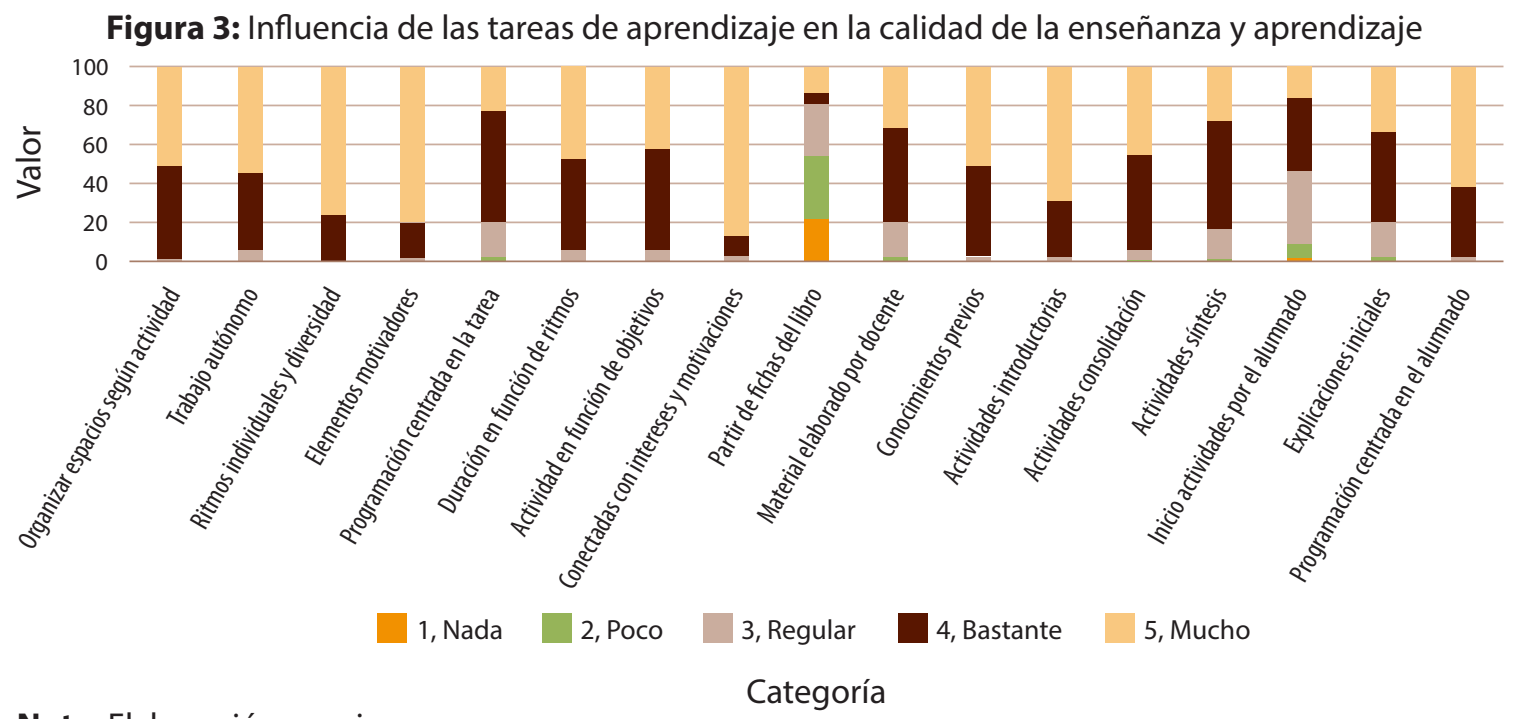


http://doi.org/10.15359/ree.24-3.11

http://www.una.ac.cr/educare

educare@una.ac.cr

En relación con la dimensión distribución del espacio, el equipo de docentes de educación infantil apuesta por espacios polivalentes y funcionales. El 48,6\% de docentes cree que influye mucho contemplar zonas de actividad para distintos tipos de agrupamiento, así como tener en cuenta las condiciones físicas y de infraestructura del aula $(43,8 \%)$. Y considera que influye bastante hacer polivalentes distintos rincones (52,4\%), transformar los espacios e instalaciones según los objetivos y necesidades del momento (49,5\%), y distribuir el mobiliario en zonas diversificadas y delimitadas (48,6\%).

Sobre la dimensión distribución del tiempo (Figura 4), el 67,6\% de docentes entiende que tiene mucha, influencia en la calidad de la enseñanza y el aprendizaje, la tarea de flexibilizar la duración de las actividades o rutinas en función de las necesidades del alumnado. También se observa un porcentaje elevado de docentes que considera muy influyente el tiempo que dedica a la preparación de los materiales, la programación, la formación y la autoevaluación (63,8\%). Por el contrario, se considera menos relevante el cumplimiento de los tiempos asignados para la realización de las tareas, al asumir la flexibilidad en la planificación de las enseñanzas.

Figura 4: Influencia de la distribución del tiempo en la calidad de la enseñanza y aprendizaje

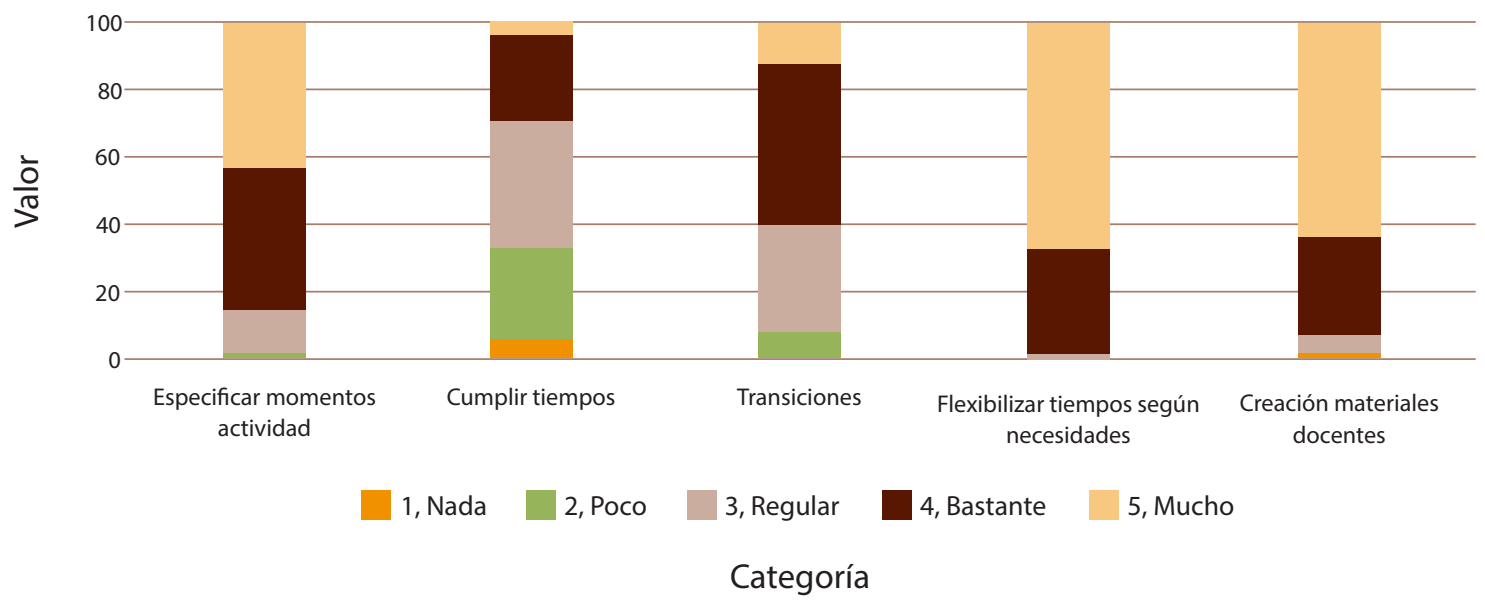

Nota: Elaboración propia.

En relación con las estrategias docentes utilizadas para gestionar el clima de aula, el equipo de docentes considera (en valores superiores al 70\%) que existen estrategias muy influyentes en la calidad de la enseñanza. Estas estrategias son las siguientes: ser receptivo o receptiva a los conflictos afectivo-emocionales y proporcionar medios para afrontarlos (70,5\%), reforzar buenos comportamientos del alumnado (83,8\%), alentar las mejoras y el esfuerzo del alumnado (83,8\%), intervenir individualmente con el alumnado que lo necesite $(76,2 \%)$, infundir sentimientos 
http://doi.org/10.15359/ree.24-3.11

de calma en clase (70,5\%), utilizar el refuerzo positivo (70,5\%), dar a conocer las normas de comportamiento y rutinas al inicio del curso (74,3\%), dar consignas claras y estables (76,2\%), crear un clima cooperativo (77,1\%) y favorecer un clima lúdico en el aula $(73,3 \%)$.

Otro de los objetivos establecidos era conocer la opinión del equipo de docentes en relación con la planificación de medios y recursos educativos (Figura 5). Al respecto, la totalidad indicó que las decisiones adoptadas y puestas en marcha sobre medios y recursos influyen en mayor o menor medida en la calidad de la enseñanza (no se observa ningún valor "1=nada" en ningún ítem). La mayoría de docentes considera que para que se produzca una enseñanza de calidad es necesario planificar con suficiente antelación los materiales que se van a necesitar (61,9\%), seleccionar materiales que permitan al estudiantado explorar y experimentar (70,5\%) pero, sobre todo, consideran muy importante la selección de materiales adecuados a la edad y a los intereses del alumnado $(75,2 \%)$ y el uso de recursos variados $(72,4 \%)$.

Figura 5: Influencia de los medios y recursos educativos

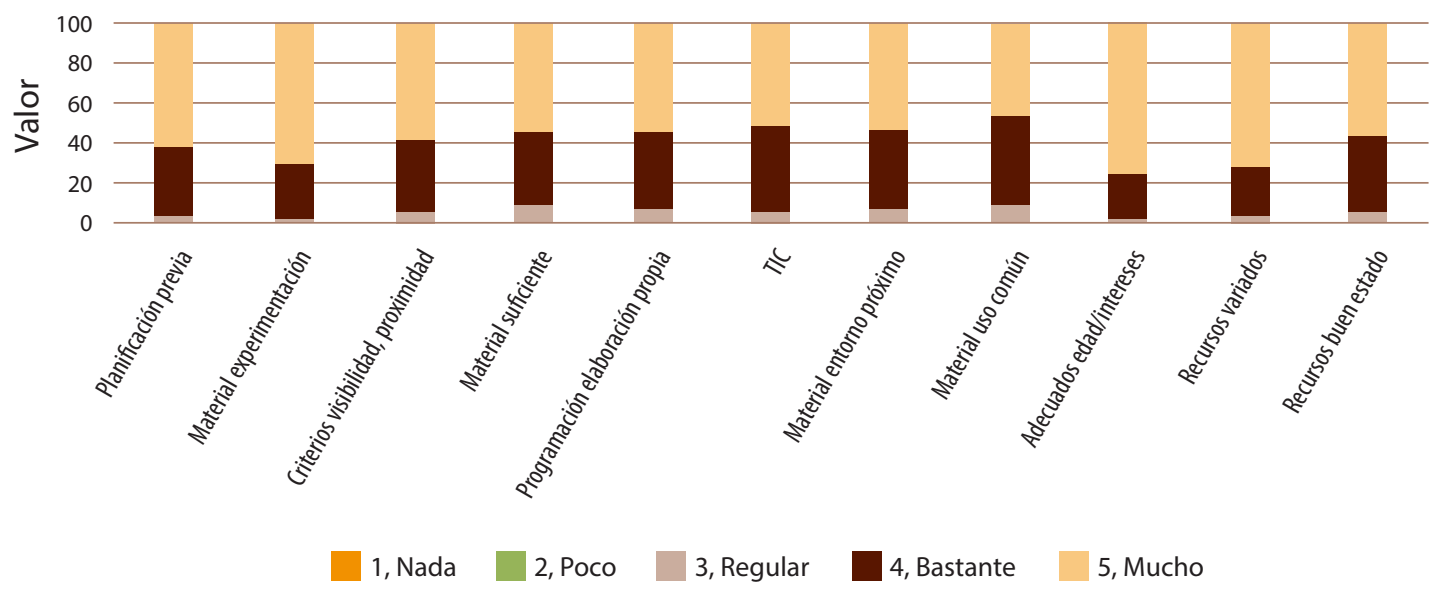

Categoría

Nota: Elaboración propia.

Finalmente, respecto a la dimensión evaluación (Figura 6), los sujetos participantes consideran que influye mucho en la calidad de la enseñanza el que se realice una primera evaluación de cada estudiante al iniciar el curso (60,0\%). Asimismo, consideran que influye mucho en esa calidad el hecho de que se registre información del alumnado al finalizar la unidad didáctica o el proyecto (54,3\%). Igualmente, creen que es necesario registrar información para la evaluación de éstos, pero que esta evaluación habría de abordarse trimestralmente 
http://doi.org/10.15359/ree.24-3.11

http://www.una.ac.cr/educare

educare@una.ac.cr

(56,2\%). Además, un 54,3\% de docentes plantean que tiene mucha importancia que se utilicen diversos instrumentos para la recogida de información como diarios, notas de campo, registros anecdóticos, audiovisuales, etc.

Figura 6: Influencia de la evaluación en la calidad de la enseñanza y aprendizaje.

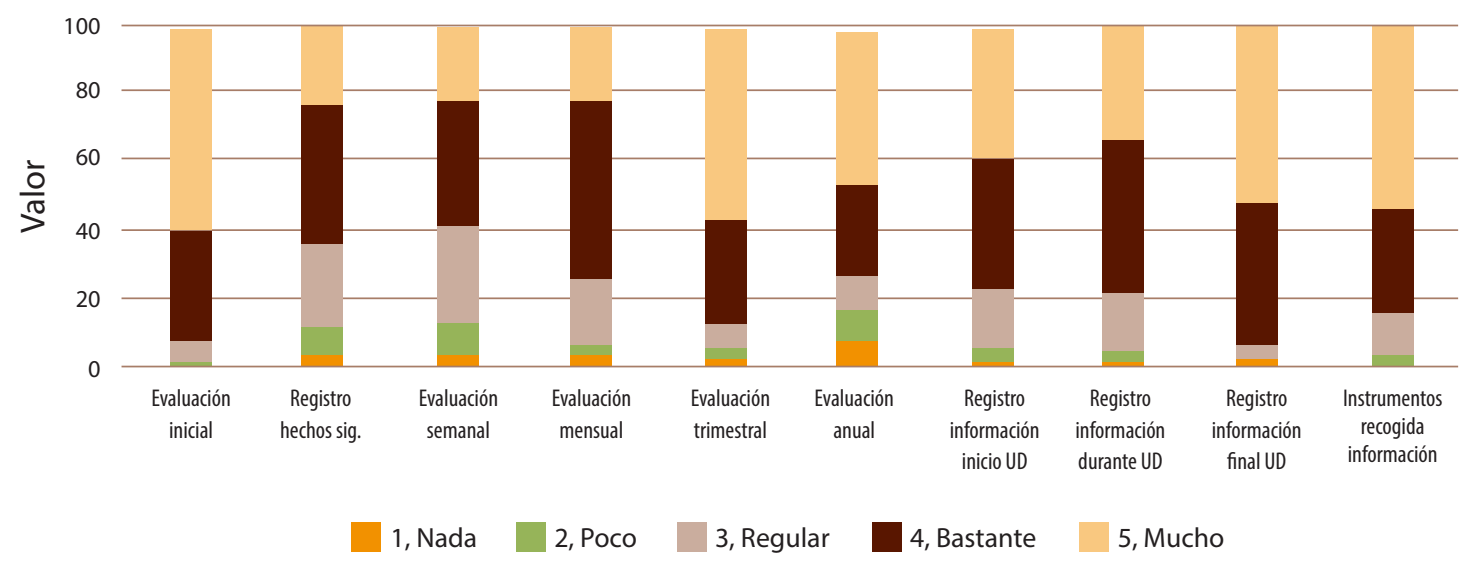

Categoría

Nota: Elaboración propia.

En líneas generales, para que los procesos evaluativos sean de calidad, se considera necesario realizar evaluaciones en diferentes momentos y utilizando diversas técnicas e instrumentos de evaluación que permitan contrastar la información registrada. Este hallazgo evidencia una concepción docente que entiende la evaluación como un proceso continuo y formativo (Vallejo Ruiz y Molina Saorín, 2014).

\section{b. Análisis correlacional de los resultados}

A continuación, se analiza si las concepciones docentes anteriormente expuestas son significativamente relevantes entre sí. Para ello, se extrajo el grado de significación (coeficiente de contingencia de Pearson) de cada uno de los ítems en función de las características personales y profesionales de la muestra; pero debido a la amplitud de información recabada, para este trabajo solo se presenta la dimensión en su totalidad, desconsiderando cada uno de los ítems que la componen. No obstante, se identifican -dentro de cada una de las dimensiones analizadas- los ítems que obtienen diferencias estadísticamente significativas.

En cuanto a la dimensión contexto y características del alumnado se observan diferencias significativas en una única variable independiente. Concretamente, como puede observarse en la Tabla 2, existe relación positiva $(p=0,014<0,05)$ entre la variable titularidad del centro al que pertenece el personal docente y las concepciones docentes sobre la influencia del contexto y las características del alumnado en la calidad del proceso de enseñanza y aprendizaje. 
http://doi.org/10.15359/ree.24-3.11

Tabla 2: Coeficiente de contingencia de la dimensión I

\begin{tabular}{lcc}
\hline & Valor & Sig. aproximada \\
\hline Género del sujeto & 0,571 & 0,766 \\
Edad del sujeto & 0,12 & 0,086 \\
Experiencia docente & 0,802 & 0,252 \\
Aula que tutela & 0,810 & 0,107 \\
Titularidad del centro & 0,772 & $\underline{\mathbf{0 , 0 1 4}}$ \\
Titulación del personal docente & 0,808 & 0,132 \\
No de estdiantes en el aula & 0,760 & 0,078 \\
\hline
\end{tabular}

Nota: Elaboración propia.

En función de la titularidad del centro destacan cuatro ítems considerados con diferencias significativas:

- Antecedentes anteriores al segundo ciclo de educación infantil.

- Variables cognitivas del alumnado (inteligencia, estrategias cognitivas, lingüísticas, etc.).

- Cualidades del alumnado (autoestima, autoeficacia, responsabilidad).

- Ausencias reiteradas.

Este hallazgo plantea que personal docente de la etapa de Educación infantil tienen una concepción ciertamente determinista en relación con las características y contexto del estudiantado y la calidad de la enseñanza y el aprendizaje.

En la dimensión II programación, no se han encontrado diferencias significativas en relación con ninguna de las características de la muestra ( $p>0,05)$. Por consiguiente, no se puede afirmar que tales características tengan una influencia determinante en las concepciones sobre la programación de los procesos de enseñanza y aprendizaje.

Como se observa en la Tabla 3, en cuanto al clima de aula (dimensión III), se encuentran diferencias significativas con respecto al título académico del personal docente $(p=0,012<0,05)$. Concretamente, puede afirmarse que existe relación entre la variable titulación de docente y su concepción sobre la influencia del clima de aula en la calidad de la enseñanza y aprendizaje en esta etapa. 
http://doi.org/10.15359/ree.24-3.11

http://www.una.ac.cr/educare

educare@una.ac.cr

Tabla 3: Coeficiente de contingencia de la dimensión III

\begin{tabular}{lcc}
\hline & Valor & Sig. Aproximada \\
\hline Género del sujeto & 0,516 & 0,075 \\
Edad del sujeto & 0,648 & 0,637 \\
Experiencia docente & 0,650 & 0,608 \\
Aula que tutela & 0,666 & 0,396 \\
Titularidad del centro & 0,582 & 0,485 \\
Titulación del personal docente & 0,719 & $\underline{\mathbf{0 , 0 1 2}}$ \\
No de estudiantes en el aula & 0,630 & 0,138 \\
\hline
\end{tabular}

Nota: Elaboración propia.

Para esta dimensión, pueden destacarse algunos ítems que presentan diferencias significativas en más de una variable independiente:

- El ítem 7.2. Ser receptivo a los conflictos afectivo-emocionales y proporcionar medios para afrontarlos muestra diferencias significativas en relación con la titulación docente $(p=0,000<0,05)$ y el número de alumnos y alumnas por aula $(p=0,029<0,05)$.

- El ítem 7.8. Reforzar los buenos comportamientos del alumnado tiene diferencias significativas en el género $(p=0,036<0,05)$, titulación docente $(p=0,003<0,05)$ y número de estudiantes por aula $(p=0,014<0,05)$.

- El ítem 7.9. Valorar, expresamente, el trabajo bien hecho manifiesta diferencias significativas en cuanto al género $(p=0,008<0,05)$ y la edad del personal docente $(p=0,047<0,05)$.

- El ítem 7.10. Alentar las mejoras y el esfuerzo de un alumno o una alumna se encuentra relacionado significativamente con la experiencia docente $(p=0,011<0,05)$ y su titulación $(p=0,004<0,05)$.

- El ítem 7.21. Establecer relaciones formales y de autoridad presenta diferencias significativas en relación con la experiencia docente $(p=0,028<0,05)$ y la titularidad del centro $(p=0,028<0,05)$.

Estas evidencias plantean cómo el refuerzo positivo al alumnado tiene una alta correlación positiva con la edad del personal docente, sus años de experiencia o género. Destaca el hallazgo de cómo se establecen tipos de relaciones diferentes según se trata de centros, públicos o privados-concertados. 
http://doi.org/10.15359/ree.24-3.11

Respecto a los recursos educativos y medios didácticos (dimensión IV), se encuentran diferencias significativas en una de las variables independientes (Tabla 4).

Tabla 4: Coeficiente de contingencia de la dimensión IV

\begin{tabular}{lcc}
\hline & Valor & Sig. Aproximada \\
\hline Género del sujeto & 0,320 & 0,684 \\
Edad del sujeto & 0,487 & 0,916 \\
Experiencia docente & 0,544 & 0,513 \\
Aula que tutela & 0,514 & 0,775 \\
Titularidad del centro & 0,500 & 0,240 \\
Titulación del personal docente & 0,576 & 0,219 \\
No de estudiantes en el aula & 0,574 & $\mathbf{0 , 0 2 4}$ \\
\hline
\end{tabular}

Nota: Elaboración propia.

Se manifiesta relación entre la ratio (número de alumnos y alumnas por aula), la titularidad del centro y las concepciones sobre la influencia de los recursos didácticos en la calidad de la enseñanza y aprendizaje $(p=0,024<0,05)$. Específicamente, pueden determinarse algunas diferencias significativas destacables en ítems de esta dimensión:

- En el ítem 8.1. Planificar con antelación el material necesario se muestran estas diferencias en relación con el género $(p=0,028<0,05)$, la titularidad del centro $(p=0,022<0,05)$ y el número de estudiantes dentro del aula $(p=0,041<0,05)$.

- El ítem 8.4. Preparar suficiente material para cada zona con objeto de que el alumnado pueda trabajar autónomamente manifiesta las diferencias significativas con respecto a la edad del personal docente $(p=0,027<0,05)$ y la titularidad del centro de pertenencia $(p=0,029<0,05)$.

- dEl ítem 8.9. Seleccionar materiales adecuados a la edad y a los intereses del alumnado presenta diferencias significativas en cuanto a la titulación docente $(p=0,006<0,05)$ y el número de estudiantes dentro del aula $(p=0,045<0,05)$.

Por último, en la dimensión de evaluación recogida en la Tabla 5, también se aprecian diferencias significativas en la variable referida a la titulación docente. 
http://doi.org/10.15359/ree.24-3.11

http://www.una.ac.cr/educare

educare@una.ac.cr

Tabla 5: Coeficiente de contingencia de la dimensión V

\begin{tabular}{lcc}
\hline & Valor & Sig. Aproximada \\
\hline Género del sujeto & 0,328 & 0,991 \\
Edad del sujeto & 0,635 & 0,783 \\
Experiencia docente & 0,698 & 0,076 \\
Aula que tutela & 0,680 & 0,226 \\
Titularidad del centro & 0,542 & 0,842 \\
Titulación del personal docente & 0,704 & $\underline{\mathbf{0 , 0 4 8}}$ \\
No de estudiantes en el aula & 0,596 & 0,521 \\
\hline
\end{tabular}

Nota: Elaboración propia.

De esta forma, se determina que existe relación entre la formación académica del personal docente y sus concepciones sobre la evaluación $(p=0,048<0,05)$. Los ítems que presentan diferencias significativas son:

- El ítem 9.3. Registrar información para la evaluación del alumnado semanalmente muestra estas diferencias según el curso que tutela el personal docente $(p=0,005<0,05)$ y titularidad del centro al que pertenece $(p=0,004<0,05)$.

- El ítem 9.5. Registrar información para la evaluación del alumnado trimestralmente presenta diferencias significativas en relación con la experiencia docente $(p=0,005<0,05)$ y la titulación docente $(p=0,041<0,05)$.

Estos resultados manifiestan cómo el registro de información sobre la evolución del alumnado (evaluación) de forma semanal; es decir, más periódica, correlaciona positivamente con la titularidad de los centros. En cambio, cuando esta información es recogida trimestralmente -como lo establece la normativa educativa estatal- la variable de titularidad de los centros no aporta diferencias estadísticamente significativas. Hallazgo ciertamente preocupante.

\section{Conclusiones del estudio}

En este artículo se describen las concepciones que tiene el personal docente de educación infantil de la Región de Murcia (España), sobre los factores que condicionan la calidad de los procesos de enseñanza y aprendizaje. Los hallazgos alcanzados ponen de manifiesto que las estrategias docentes para gestionar el clima de aula y las decisiones relacionadas con los medios y recursos educativos son los factores más condicionantes de esta calidad. Estos resultados 
http://doi.org/10.15359/ree.24-3.11

fuerzan la tesis de Téllez Tinoco (2014) sobre el valor de crear ambientes para intensificar el aprendizaje del alumnado, o de Ruiz Gutiérrez y Rebollo Aranda (2015) quienes otorgan al personal docente la responsabilidad de generar escenarios ricos y estimulantes que permitan al estudiantado explorar y vivenciar situaciones de aprendizaje. Asimismo, son concordantes con el estudio de Murillo Torrecilla et al. (2011) sobre cómo la selección de determinados materiales y recursos educativos posibilitan la creación de escenarios ricos de aprendizaje y de experimentación para el alumnado.

Por otro lado, cabe destacar algunas de las concepciones profesionales del personal docente de educación infantil: para estos grupos docentes es trascendental la implicación y participación de los padres y madres en los procesos de enseñanza y aprendizaje. El equipo de docentes de esta etapa educativa manifiesta que uno de los factores fundamentales para la calidad de los procesos de enseñanza y aprendizaje es la participación activa de las familias. También consideran que la programación y planificación de estos procesos deben tener en cuenta, en primera instancia, las necesidades, capacidades, intereses y motivación del alumnado. Además, este profesorado enfatiza, claramente, en la necesaria atención a la diversidad, tanto en la planificación de objetivos y contenidos como en el diseño de tareas y actividades.

Por otro lado, conciben la gestión del clima de aula como un elemento vinculado a ciertas acciones que permiten el desarrollo adecuado de los procesos de enseñanza y aprendizaje; destacando, fundamentalmente, el uso del refuerzo (sobre todo positivo) ante los comportamientos del alumnado.

Como aspectos que debilitan la calidad de los procesos de enseñanza y aprendizaje se ha identificado las faltas de asistencia reiteradas del alumnado. El personal docente manifiesta, en este caso, que dichas ausencias pueden debilitar los procesos de aprendizaje del alumnado $y$, en consecuencia, la calidad de la educación que reciben. Consideramos que este factor se encuentra relacionado directamente con la implicación de las familias que también es percibido como un aspecto negativo en caso de no producirse.

Para finalizar, se resalta el hallazgo relativo a la correlación positiva entre calidad de la enseñanza y aprendizaje, y las características personales y familiares del estudiantado. Si bien es cierto que infinidad de estudios ratifican esta correlación (Córdoba et al., 2011; Jiménez Morales y López-Zafra, 2009; Lastre Meza et al., 2018), consideramos poco beneficioso para los niños y niñas de edades comprendidas entre 3-6 años, que sus docentes trabajen desde esta concepción preestablecida. Estamos plenamente convencidas de que la etapa de educación infantil ha de ser una etapa llena de fantásticas experiencias de aprendizaje que, en ningún caso, han de quedar mediatizadas por preconcepciones docentes sobre el potencial de estos niños y niñas que se encuentran en pleno desarrollo. Estas preconcepciones solo pueden limitar o plantear sesgos sobre los alcances de estos menores y, por tanto, aunque sea de manera inconsciente, condicionar la práctica docente. 
http://doi.org/10.15359/ree.24-3.11

http://www.una.ac.cr/educare

educare@una.ac.cr

Como futuras líneas de investigación, se hace necesaria la prioridad de ahondar en las conceptualizaciones sobre la práctica docente, analizando -por ejemplo- si las percepciones docentes sobre su desempeño profesional (en su aplicación práctica) mantienen correspondencias biunívocas con el ejercicio práctico de su actuación profesional habitual, o si -por el contrario- merecen el análisis pormenorizado de determinado tipo de variables que, con toda probabilidad, bien pudieran incidir en la configuración no solo de esa percepción sobre el desempeño, sino también (y lo que se deriva de lo anterior) incidir directamente en la actuación práctica como profesionales de la educación (tal y como anuncian autores como Barrón Tirado, 2015; Capellini y Rodrigues, 2009; Lucas, 2009; Malcolm, 2006; Solis, 2015).

\section{Referencias}

Aguerrondo, I. (2014). La calidad de la educación: Ejes para su definición y evaluación. https:// www.researchgate.net/publication/44818477

Barrón Tirado, C. (2015). Concepciones epistemológicas y práctica docente. Una revisión. REDU. Revista de Docencia Universitaria, 13(1) 35-56. https://doi.org/10.4995/redu.2015.6436

Capellini, V. L. M. F. y Rodrigues, O. M. P. R. (2009). Concepções de professores acerca dos fatores que dificultam o processo da educação inclusiva. Educação, 32(3), 355-364. http:// revistaseletronicas.pucrs.br/ojs/index.php/faced/article/view/5782/4203

Cejudo, J., Díaz, M. V., Losada, L. y Pérez-González, J. C. (2016). Necesidades de formación de maestros de Infantil y Primaria en atención a la diversidad. Bordón, Revista de Pedagogía, 68(3), 23-39. https://doi.org/10.13042/Bordon.2016.68402

Córdoba Caro, L. G., García Preciado, V., Luengo Pérez, L. M., Vizuete Carrizosa, M. y Feu Molina, S. (2011). Determinantes socioculturales: Su relación con el rendimiento académico en alumnos de enseñanza secundaria obligatoria. Revista de Investigación Educativa, 29(1), 83-96. https://revistas.um.es/rie/article/view/110361

Devlin, M. (2006). Challenging accepted Wisdom about the place of conceptions of teaching in university teaching improvement. International Journal of Teaching and Learning in Higher Education, 18(2), 112-119. http://dro.deakin.edu.au/eserv/DU:30006685/Devlinchallenging accepted.pdf

Hernández Sampieri, R., Fernández Collado, C. y Baptista Lucio, P. (2014). Metodología de la investigación (6. ed.). McGrawHill.

Iglesias Forneiro, M. L. (2008). Observación y evaluación del ambiente de aprendizaje en educación infantil: Dimensiones y variables a considerar. Revista Iberoamericana de Educación, 47, 49-70. https://rieoei.org/historico/documentos/rie47a03.htm 
Jiménez Morales, M. I. y López-Zafra, E. (2009). Inteligencia emocional y rendimiento escolar: Estado actual de la cuestión. Revista Latinoamericana de Psicología, 41(1), 69-79. https:// www.redalyc.org/articulo.oa?id $=80511492005$

Lastre Meza, K., López Salazar, L. D. y Alcázar Berrío, C. (2018). Relación entre apoyo familiar y el rendimiento académico en estudiantes colombianos de educación primaria. Psicogente, 21(39), 102-115. http://doi.org/10.17081/psico.21.39.2825

Latorre, A., del Rincón, D. y Arnal, J. (2003). Bases metodológicas de la investigación educativa. Ediciones Experiencia.

Lebrero Baena, M. P. y Fernández Pérez, M. D. (2009). Algunos indicadores de calidad en la educación infantil. Teoría de la Educación. Revista Interuniversitaria, 21(2), 195-225. https:// revistas.usal.es/index.php/1130-3743/article/view/7156/7221

Lera, M. J. y Oliver, R. (2002). Escala de evaluación de contextos educativos infantiles. Universidad de Sevilla. http://www.psicoeducacion.eu/psicoeducacion/ECERS-R.pdf

Lucas, M. A. O. F. (2009). Os processos de alfabetização e letramento na educação infantil: Contribuições teóricas e concepções de profesores [Tesis de doctorado]. Universidad de São Paulo, Brasil. https://teses.usp.br/teses/disponiveis/48/48134/tde-24092009-133850/ publico/MariaAngelicaFrancisco.pdf

Malcolm, E. (2006). Teachers' conceptions of teaching, and the making of specific decisions in planning to teach. Higher Education, 51(2), 191-214. https://doi.org/10.1007/s10734-004$\underline{6382-9}$

Martínez Bonafé, J. (1988). Pensamiento del profesor y renovación pedagógica. Investigación en la escuela, 4, 13-18. https://idus.us.es/handle/11441/59109

Martínez Ortiz, M. F., Nieto Cano, J. M. y Vallejo Ruiz, M. (2016). Buenas prácticas de enseñanza desde la concepción del profesorado. EA, Escuela Abierta, 19(1), 81-100. https://doi. org/10.29257/EA19.2016.06

Miranda Esquer, J. F. y Miranda Esquer, J. B. (2012). Reflexiones sobre la calidad de la educación y sus referentes: El caso de México. Educere, 16(53), 43-52. http://www.redalyc.org/articulo. oa?id=35623538006

Murillo Torrecilla, F. J., Martínez Garrido, C. A. y Hernández Castilla, R. (2011). Decálogo para una enseñanza eficaz. REICE: Revista Electrónica Iberoamericana sobre Calidad, Eficacia y Cambio en Educación, 9(1), 6-27. http://www.redalyc.org/pdf/551/55118790002.pdf

Ruiz Gutiérrez, S. y Rebollo Aranda, M. S. (2015). El aula de educación infantil: El aula creativa. Ensayos: Revista de la Facultad de Educación de Albacete, 30(2), 71-84. https://revista.uclm. es/index.php/ensayos/article/view/647 
http://doi.org/10.15359/ree.24-3.11

http://www.una.ac.cr/educare

educare@una.ac.cr

Ruiz de Miguel, C. (2002). Propuesta y validación de un modelo de calidad en educación infantil [Tesis de doctorado]. Universidad Complutense de Madrid, España. https://eprints.ucm. es/4661/

Sammons, P., Sylva, K., Hall, J., Siraj, I., Melhuish, E., Taggart, B.y Mathers, S. (2017). Establishing the effects of quality in early childhood: Comparing evidence from England. Early Education.

Sañudo Guerra, L. y Sañudo Guerra, M. I. (2014). Las concepciones explícitas sobre evaluación en la práctica docente en educación preescolar en Jalisco, México. Revista Iberoamericana de Evaluación Educativa, 7(1), 31-42.https://revistas.uam.es/index.php/riee/article/view/3384

Solis, C. A. (2015). Creencias sobre enseñanza y aprendizaje en docentes universitarios: Revisión de algunos estudios. Propósitosy Representaciones, 3(2), 227-260. https://doi.org/10.20511/ pyr2015.v3n2.83

Soto-Grant, A. (2018). Habilidades y estrategias didácticas necesarias para la alfabetización visual en educación preescolar. Revista Electrónica Educare, 22(3), 1-17. https://doi.org/10.15359/ ree.22-3.2

Téllez Tinoco, N. (2014). Ensayo sobre el diseño de ambientes de aprendizaje. Vida Científica. Boletín científico de la escuela de preparatoria No. 4, 2(3), https://www.uaeh.edu.mx/scige/ boletin/prepa4/n3/e3.html

Vallejo Ruiz, M., y Molina Saorín, J. (2014). La evaluación auténtica de los procesos educativos. Revista Iberoamericana de Educación, 64, 11-25. https://doi.org/10.35362/rie640403

Zabalza Beraza, M. A. (1996). Calidad en la educación infantil. Narcea.

Zabalza Beraza, M. A. (2012). El estudio de las "buenas prácticas" docentes en la enseñanza universitaria. REDU. Revista de Docencia Universitaria, 10(1), 17-42. https://doi.org/10.4995/ redu.2012.6120 